\title{
A Proposed Stereochemical Mechanism for the Improved Preparation of Maleic Anhydride Cycloadduct of CLA
}

\author{
Jieyu He ${ }^{1 *}$, Jiashu Liao², Junyan Qu1 \\ ${ }^{1}$ College of Marine Science \& Technology, Hainan Tropical Ocean University, Sanya, China \\ ${ }^{2}$ College of Chemistry \& Chemical Engineering, Hunan Institute of Science \& Technology, Yueyang, China \\ Email: ^jyhe555@163.com
}

How to cite this paper: He, J.Y., Liao, J.S. and Qu, J.Y. (2021) A Proposed Stereochemical Mechanism for the Improved Preparation of Maleic Anhydride Cycloadduct of CLA. Computational Chemistry, 9, 144-160. https://doi.org/10.4236/cc.2021.93009

Received: May 29, 2021

Accepted: July 11, 2021

Published: July 14, 2021

Copyright $\odot 2021$ by author(s) and Scientific Research Publishing Inc. This work is licensed under the Creative Commons Attribution International License (CC BY 4.0).

http://creativecommons.org/licenses/by/4.0/ (c) (i) Open Access

\begin{abstract}
The fatty acid derivatives, prepared from renewable natural oils, can be used as highly promising and potential substitutes for petrochemicals. The study of process improvement and stereochemical mechanism for preparing these derivatives would be beneficial for their industrial production. Conjugated linoleic acid (CLA) containing 9cis-11trans $(9 c, 11 t)$ and 10trans-12cis (10t, 12c) isomers was prepared from Salicornia herbacea seed oil. Maleic anhydride cycloadduct of CLA (MAC) was prepared by an improved process, and it was characterized by FTIR, ${ }^{1} \mathrm{H}$ and ${ }^{13} \mathrm{C}$ NMR, etc. A new method to calculate conformers-ratio of CLA or MAC was also developed. Furthermore, the stereochemical mechanism for the improved preparation of MAC was proposed primarily by the calculation method above. The following observations were made: 1) The yield of MAC could reach as high as $96.7 \%$ under mild reaction conditions and with an easy and efficient product separation; 2) The trans-trans CLA in the $s$-cis conformation acted as a predominant reactant to Diels-Alder $[4+2]$ cycloaddition of maleic anhydride, which was the main reaction occurred simultaneously with catalytic configurational isomerizations of CLA in one step; 3) From all studied CLA conformers, the most stable conformation was the $s$-trans conformation of trans-trans CLA, while the $s$-cis conformation of trans-trans CLA had the most favorable structural parameters for cyclohexenyl ring formation; 4) Four MAC conformers derived from $9 c, 11 t$ - and $10 t, 12 c$-CLA, were obtained as final main products that were determined to be cis-cycloadducts; 5) The endo/exo ratios of the ciscycloadducts derived from $9 c, 11 t$ - and $10 t, 12 c$-CLA, were 2.14:1 and 1.99:1, respectively; and 6) The results obtained from the calculation method above were in excellent accordance with those from our experiments.
\end{abstract}




\section{Keywords}

Maleic Anhydride Cycloadducts, Improved Preparation, Conjugated Linoleic Acid, Stereochemical Mechanism, Conformers-Ratio Calculation, Conformational Isomerization

\section{Introduction}

As a green and renewable alternative to petroleum-based derivatives, MAC is a promising organic polyacid that can be widely used in the production of resin additives, complexing agents, surfactants, corrosion inhibitors, etc [1] [2] [3]. Traditionally, three independent steps were required to prepare MAC. The first step was the conjugation isomerization of linoleic acid from vegetable oils, by which CLA containing cis-trans or/and trans-cis isomer(s) was prepared; the configurational isomerization of CLA into trans-trans form by a catalyst was carried out during the second step, and the synthesis of MAC by Diels-Alder cycloaddition of maleic anhydride with the trans-trans CLA was performed during the third step. A one-pot method (i.e., direct reaction of linoleic acid with maleic anhydride) for preparing MAC was reported by Watanabe et al. [4], however, a low yield of $58 \%$ under high temperature and high pressure was observed. Afterwards, microwave-assisted organic synthesis and continuous-flow techniques to prepare MAC were employed with a relatively higher yield of $86 \%$ [5]. To the best of our knowledge, no attempt has been made to prepare MAC by the improved process, as well as to propose the stereochemical mechanism by some calculation methods.

In this work, CLA was prepared by using Salicornia herbacea seed oil as a starting material, due to the high content of linoleic acid in this oil. Besides, several improvements for the preparation of MAC were developed. For example, the latter two steps described in the traditional processes (i.e., configurational isomerization and Diels-Alder cycloaddition) were merged, and a facile oil-bath approach under mild reaction conditions (e.g., lower temperature and atmospheric pressure) was carried out. Thereby MAC could be prepared in this one-step procedure without any additional solvent or pre-transformation of as-prepared CLA into trans-trans form. In addition, the product separation procedures were improved as well; e.g., the rapid and simultaneous separation of the spent catalyst and residual CLA from reaction mixtures could be achieved by using $n$-octane as the selective solvent.

To further study the stereochemical mechanism for the improved preparation of MAC, a new method for calculation of conformers-ratio of CLA or MAC in the stereochemical reactions was developed based on Maxwell-Boltzmann statistics [6] [7]. The influences of structural parameters, electron-donor abilities and stabilities of CLA conformers on the stereochemical reactions were investigated by the developed calculation method, FMO theory [8] and Mopac-AM1 
algorithm in Chem3D Ultra 10.0 [9] [10]. The molecular structure of MAC was correctly established firstly. Besides, the two key factors influencing the stereochemical reactions in the preparation of MAC, reaction temperature and catalyst dosage, were also investigated in this work.

\section{Materials and Methods}

\subsection{Materials}

All chemicals and solvents used in this work were of analytical grade and purchased from Sinopharm Chemical Reagent CO., Ltd (Shanghai, China). Salicornia herbacea seed oil (containing $77.2 w t \%$ of linoleic acid) from Leizhou Peninsula, China, was provided by College of Life Sciences \& Ecology at our university. Methyl linoleate was prepared by the transesterification of Salicornia herbacea seed oil with methanol for determining the percentage of linoleic acid by gas chromatography (GC).

\subsection{Preparation and GC Analysis of CLA}

By using Salicornia herbacea seed oil as a starting material, CLA was prepared according to the previously reported method [11]. Ethylene glycol (50.0 g) and potassium hydroxide $(10.0 \mathrm{~g})$ were added to a $250 \mathrm{~mL}$ of round-bottom flask under nitrogen $\left(\mathrm{N}_{2}\right)$ atmosphere $\left(\mathrm{N}_{2}\right.$ gas was blown at a rate of $\left.30 \mathrm{~mL} \cdot \mathrm{min}^{-1}\right)$. The reaction mixture was stirred continuously at $160^{\circ} \mathrm{C}$ until all potassium hydroxide was dissolved. Then Salicornia herbacea seed oil (25.0 g) was added into the mixture and the reaction was heated up to $180^{\circ} \mathrm{C}$ for $4.0 \mathrm{hrs}$. After the reaction was completed, the mixture was cooled to $80^{\circ} \mathrm{C}$ and then acidified with hydrochloric acid $(\mathrm{pH}<2)$, and extracted with $100 \mathrm{~mL}$ of hexane. The hexane extract was washed to neutral with double distilled water. Anhydrous sodium sulfate was added to remove residual water. CLA was obtained by vacuum distillation.

The percentages of CLA isomers were determined by GC, which was performed on a Shimadzu GC-2010/AOC-20i. The chromatographic conditions were selected as follows: FID detector; chromatographic column, DB-WAX $(20 \mathrm{~m} \times 0.25 \mathrm{~mm}$ $\times 0.25 \mu \mathrm{m}$ ); temperature programmed: column temperature was kept constant at $140^{\circ} \mathrm{C}$ for $4 \mathrm{~min}$, then increased to $230^{\circ} \mathrm{C}$ at a rate of $30^{\circ} \mathrm{C} / \mathrm{min}$ and kept constant for $20 \mathrm{~min}$; carrier gas $\left(\mathrm{N}_{2}\right)$ flow-rate $1.28 \mathrm{~mL} / \mathrm{min}$; pressure $128 \mathrm{kPa}$; sample size $2 \mu \mathrm{L}$; and split ratio $1: 30$. The two major isomers, $9 c, 11 t$ and $10 t, 12 c$, in as-prepared CLA, were quantitatively determined by GC. And $9 \mathcal{c}, 11 t$ isomer accounted for $63.82 w t \%$.

\subsection{Improved Preparation of MAC}

An improved process for preparing MAC was carried out. CLA (14.85 g, about $0.05 \mathrm{~mol}$ of carboxyl group), maleic anhydride $(5.88 \mathrm{~g}, 0.06 \mathrm{~mol})$ and iodine $(0.04 \mathrm{~g}, 0.31 \mathrm{mmol})$ were added to a $100 \mathrm{~mL}$ of round-bottom flask under nitrogen $\left(\mathrm{N}_{2}\right)$ atmosphere $\left(\mathrm{N}_{2}\right.$ gas was blown into the reaction at a rate of $\left.15 \mathrm{~mL} \cdot \mathrm{min}^{-1}\right)$. 
The mixture was evenly dispersed under stirring and heated up to $60^{\circ} \mathrm{C}$ for 4.0 hrs in an oil-bath. After the reaction was completed, $50 \mathrm{~mL}$ of $n$-octane was added into the reaction; and the mixture was stirred to obtain a homogeneous dispersion. After being cooled to the room temperature, the mixture was filtrated to remove the solvent. The residual solid on the filter paper was collected and dissolved in $50 \mathrm{~mL}$ of diethyl ether, which was washed using deionized water until the filtrate became neutral. A white solid of MAC (approx. 18.3 g) could be obtained by removing ether via distillation. The crude product was purified by recrystallization from petroleum ether/diethyl ether (volume ratio of 4:1). Melting point: $56.5^{\circ} \mathrm{C}$; Elemental analysis $\left(\mathrm{C}_{22} \mathrm{H}_{34} \mathrm{O}_{5}\right)$ : measured value (calculated value, \%): C, 69.90 (69.81); H, 9.08 (9.06).

\subsection{FTIR and NMR Characterization of MAC}

FTIR spectrum was recorded on a Shimadzu FTIR-8400S spectrophotometer in the range of 400 to $4000 \mathrm{~cm}^{-1}$ by the $\mathrm{KBr}$ pellet pressed technique. ${ }^{1} \mathrm{H}$ and ${ }^{13} \mathrm{C}$ NMR spectra were recorded on a Bruker AV 400 NMR spectrometer in $\mathrm{CDCl}_{3}$; and chemical shifts were referenced to an internal standard tetramethylsilane (TMS).

\section{Results and Discussions}

\subsection{Effects of Reaction Temperature on Yield of MAC}

The reaction temperature played a decisive role in the preparation of MAC by CLA. The yields of MAC under different reaction temperatures were shown (Figure 1). The conditions for the improved preparation of MAC were optimized as follows: molar ratio of CLA to maleic anhydride: 1:1.2, reaction time: $4.0 \mathrm{hrs,}$ and catalyst dosage: $0.2 w t \%$.

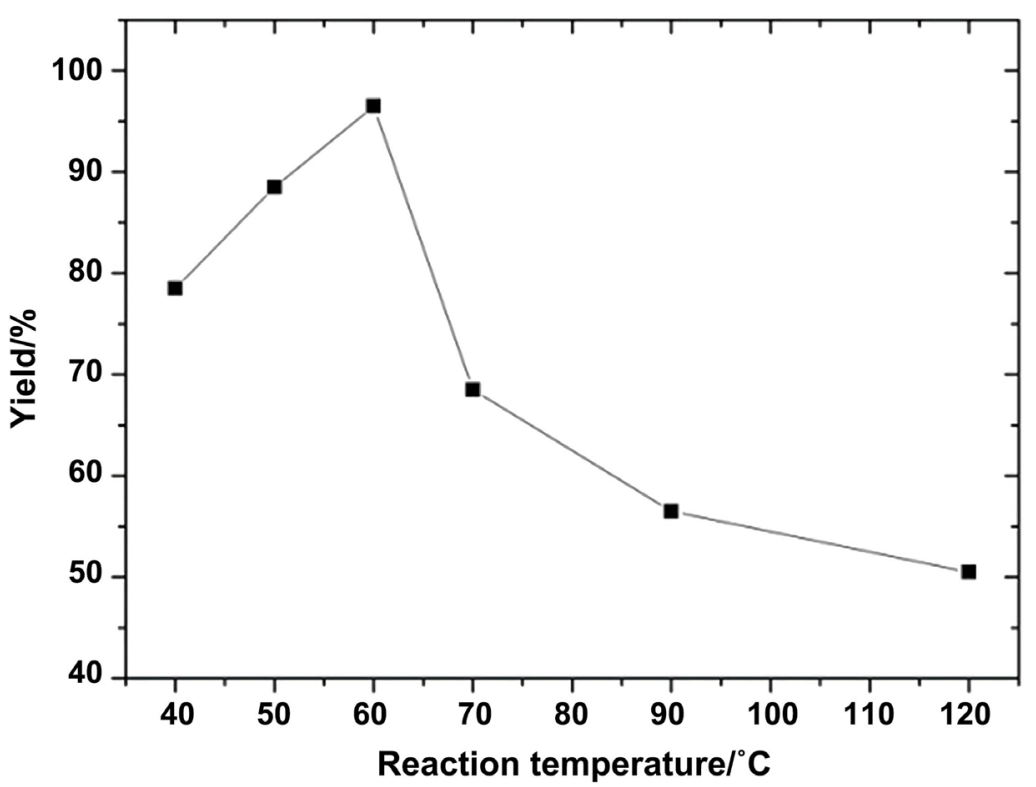

Figure 1. Effects of different reaction temperatures on yield of MAC. 
It can be seen from Figure 1 that the yield of MAC increased with an increase in the reaction temperature range $40^{\circ} \mathrm{C}$ to $60^{\circ} \mathrm{C}$, and the highest yield of 96.7 $w t \%$ was obtained at $60^{\circ} \mathrm{C}$. Then the yield decreased as the reaction temperature increased from $60^{\circ} \mathrm{C}$ to $120^{\circ} \mathrm{C}$, indicating that the cycloaddition reaction was thermally reversible, and high reaction temperature was beneficial to the reverse reaction. Therefore, the cycloaddition reaction was carried out at $333.15 \mathrm{~K}$ (i.e., $60^{\circ} \mathrm{C}$ ); and a high yield of $96.7 w t \%$ could be obtained for MAC, which was much higher than the results previously reported.

\subsection{Effects of Catalyst Dosage on Yield of MAC}

The cycloaddition of maleic anhydride could form a normal Diels-Alder adduct, which could be greatly facilitated if CLA has a trans-trans form [12]. Many methods available for the isomerization of CLA into trans-trans form from the cis-trans and trans-cis form were reported (e.g., p-toluene sulfonic acid-catalyzed, sulfur powder-catalyzed), while iodine was chosen as a catalyst in this work because side reactions and undesired double-bond migration could be minimized [13]. The yields of MAC under different dosages of iodine catalyst were shown (Figure 2). The conditions were set as follows: molar ratio of CLA to maleic anhydride: $1: 1.2$, reaction time: $4.0 \mathrm{hrs}$; and reaction temperature: $60^{\circ} \mathrm{C}$.

It can be seen from Figure 2 that the yield of MAC could reach as high as $96.7 \%$ at the catalyst dosage of $0.2 w t \%$ (versus the total mass of the reactants). No desired product would be obtained in the absence of the catalyst, iodine, while a solid gel would be formed. This may be attributed to the fact that polymerization reaction, rather than Diels-Alder cycloaddition, would occur in the absence of iodine. That is to say, the $[4+2]$ cycloaddition reaction between trans-trans CLA and maleic anhydride occurred due to the catalytic properties of configurational isomerization of CLA [14]. In addition, fewer yields would be obtained when

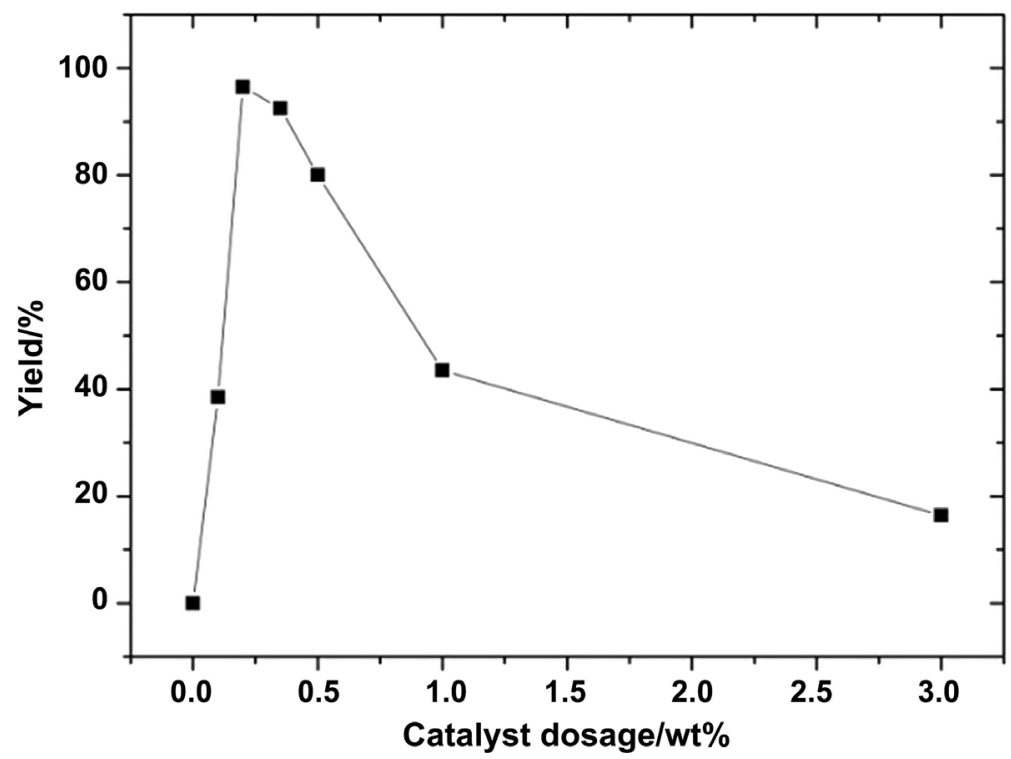

Figure 2. Effects of different catalyst dosages on yield of MAC. 
catalyst dosage increased from 0.2 to $3.0 w t \%$, which may be due to the possible addition reaction between CLA and excessive iodine. Therefore, a small quantity of iodine was employed to induce the isomerization of cis-trans and trans-cis into trans-trans form during simultaneous cycloaddition of maleic anhydride. Nevertheless, there were too many different conformers for the same trans-trans CLA, and which conformations (-ratios) of desired product molecules would be generated from their cycloaddition reactions should be clearly demonstrated. Therefore, it is important to deeply investigate the possible stereochemical mechanism for the cycloaddition reaction abovementioned.

\subsection{FTIR Analysis of MAC}

FTIR spectrum of MAC purified by recrystallization was shown as Figure 3.

It can be seen from Figure 3 that main absorption peaks were found as follows. The peaks at $3027.8,1637.0$ and $726.9 \mathrm{~cm}^{-1}$ could be attributed to $v(=\mathrm{C}-\mathrm{H})$, $v(\mathrm{C}=\mathrm{C})$ and $\omega(=\mathrm{C}-\mathrm{H})$ of cyclohexenyl group, respectively. The peaks at 1848.0, 1783.1 and $1192.3 \mathrm{~cm}^{-1}$ corresponded to $v_{\mathrm{s}}(\mathrm{C}=\mathrm{O}), v_{\mathrm{as}}(\mathrm{C}=\mathrm{O})$ and $v(\mathrm{C}-\mathrm{O})$ of pentacyclic dianhydride group, respectively. And the peaks at $2500.0-3550.0,1703.5$, and $939.8 \mathrm{~cm}^{-1}$ could be assigned to $v(\mathrm{O}-\mathrm{H}), v(\mathrm{C}=\mathrm{O})$ and $\delta(\mathrm{C}-\mathrm{OH})$ of carboxyl group, respectively. The presence of cyclohexenyl group confirmed that $\mathrm{Di}$ els-Alder [4+2] cycloaddition was carried out.

\section{4. ${ }^{1} \mathrm{H}$ NMR Analysis of MAC}

${ }^{1} \mathrm{H}$ NMR spectrum of the purified MAC was shown as Figure 4.

The specific analysis of Figure 4 was as follows. ${ }^{1} \mathrm{H}$ NMR $\left(\mathrm{CDCl}_{3}, 400 \mathrm{MHz}\right)$ ppm: $5.82(\mathrm{~s}, 2 \mathrm{H}), 3.36-3.35(\mathrm{dd}, J=4.5 \mathrm{~Hz}, 2 \mathrm{H}), 2.37-2.34(\mathrm{t}, J=7.5 \mathrm{~Hz}, 2 \mathrm{H})$, $2.24-2.23(\mathrm{~m}, 2 \mathrm{H}), 1.92-1.81(\mathrm{~m}, 2 \mathrm{H}), 1.80-1.74(\mathrm{~m}, 2 \mathrm{H}), 1.66-1.63(\mathrm{q}, J=7$

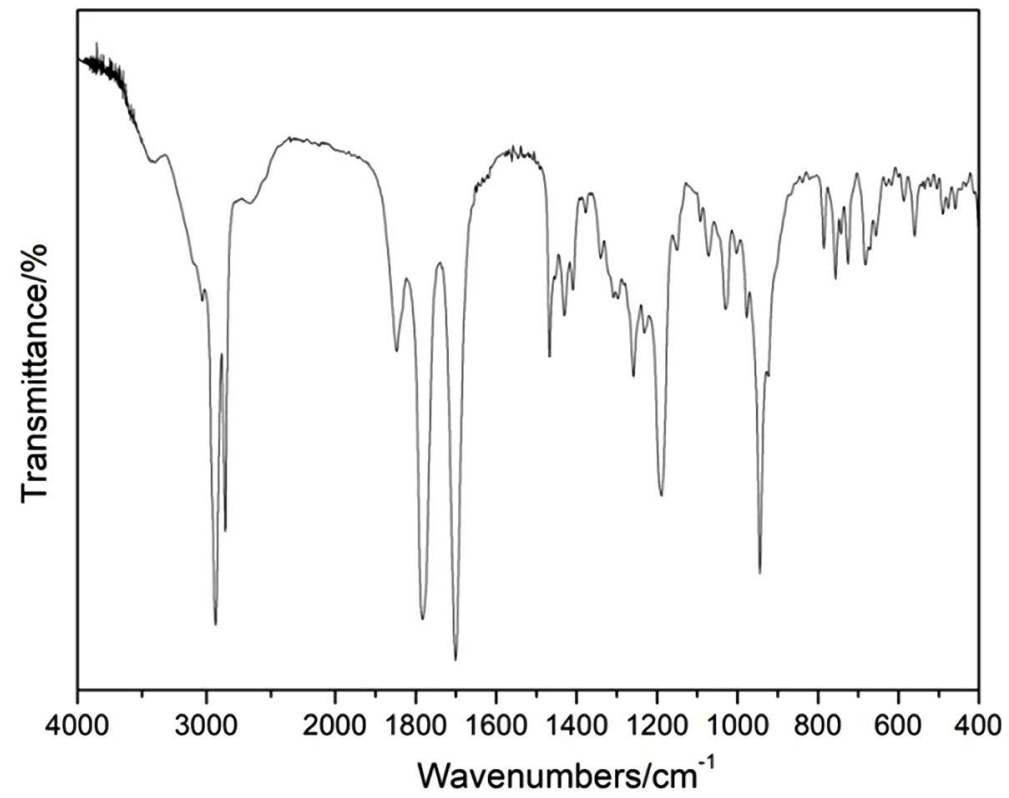

Figure 3. FTIR spectrum of MAC. 

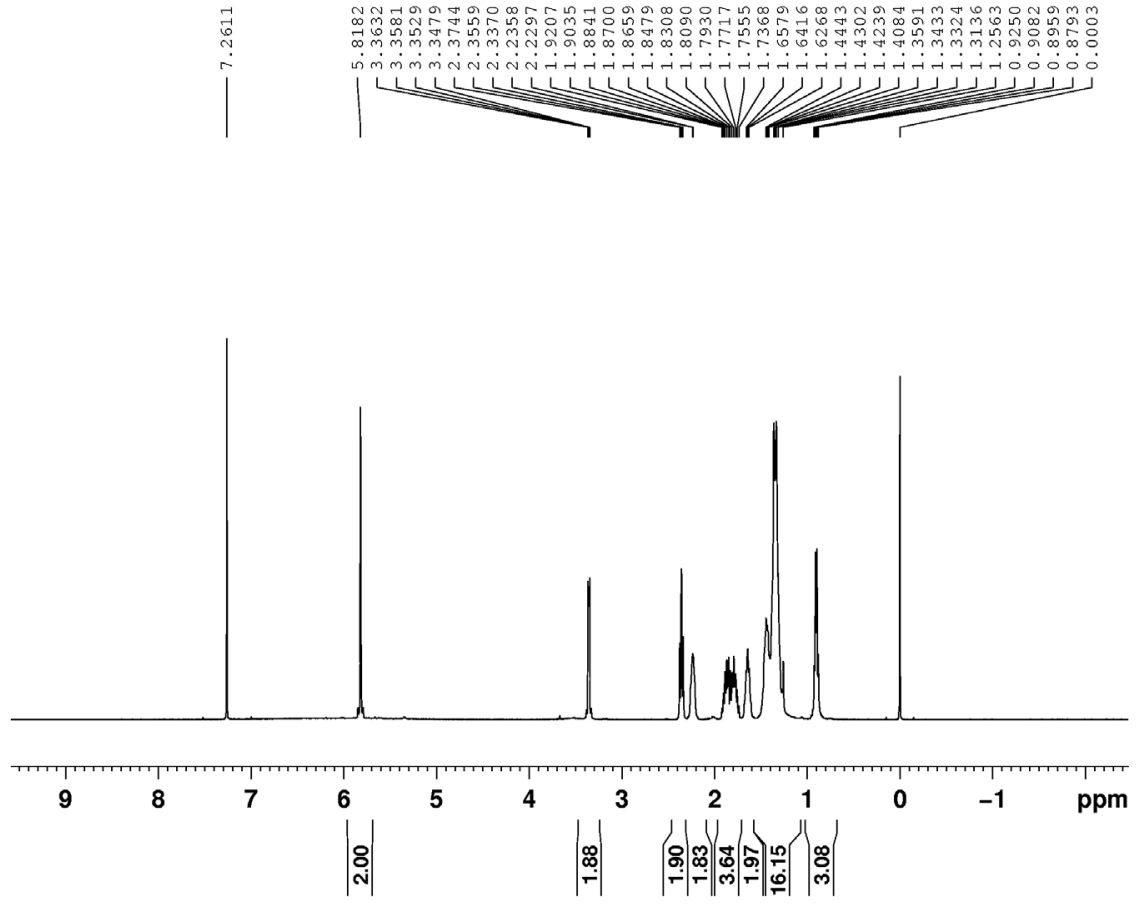

Figure 4. ${ }^{1} \mathrm{H}$ NMR spectrum of MAC.

$\mathrm{Hz}, 2 \mathrm{H}), 1.44-1.41(\mathrm{~m}, 4 \mathrm{H}), 1.36-1.33(\mathrm{~m}, 8 \mathrm{H}), 1.31-1.26(\mathrm{~m}, 4 \mathrm{H})$, and $0.93-$ $0.88(\mathrm{t}, J=7 \mathrm{~Hz}, 3 \mathrm{H})$. The result of ${ }^{1} \mathrm{H}$ NMR analysis was consistent with that of FTIR.

\section{5. ${ }^{13} \mathrm{C}$ NMR Analysis of MAC}

${ }^{13} \mathrm{C}$ NMR spectrum of the purified MAC was given as Figure 5.

The attribution of the ${ }^{13} \mathrm{C}$ NMR chemical shifts in Figure 5 was as follows. ${ }^{13} \mathrm{C}$ NMR ( $\left.\mathrm{CDCl}_{3}, 100 \mathrm{MHz}\right)$ ppm: $178.9(\mathrm{COOH}), 171.8$ and 171.7 (C-1' and C-3'), 132.9 and 132.8 (C-5' and C-6'), 44.3 (C-3'a), 44.2 (C-7'a), 37.5 (C-4'), 37.4 (C-7'), 34.0 (C-2), 32.2 (C-4"), 31.7(C-8), 30.1 (C-1"), 29.7 (C-3"), 29.3 (C-5), 29.1 (C-6), 29.0 (C-4), 27.6 (C-7), 27.4 (C-2"), 24.7 (C-3), 22.7 (C-5"), and 14.1(C-6"). Accordingly, MAC molecular structure illustrated in Figure 5 was obtained.

\subsection{Stereochemical Mechanism for the Preparation of MAC}

\subsubsection{Proposed Stereochemical Reactions}

Based on the experimental results, the possible stereochemical reactions in the preparation of MAC are proposed (Scheme 1).

As can be seen from Scheme 1, four stereochemical reactions are involved in the preparation of MAC: 1) constitutional isomerization of linoleic acid, i.e., conjugated isomerization Equation $(1) ; 2$ ) configurational isomerization of CLA, i.e., cis/ trans isomerization Equation (4) and Equation (5); 3) conformational isomerization of conjugated dienes of CLA, i.e., isomerization of conformer 1, 2, 6 and 7 into conformer 3, 4, 9 and 10, respectively; and 4) cycloaddition reaction, e.g., Diels-Alder reaction Equation (8) and Equation (9). 


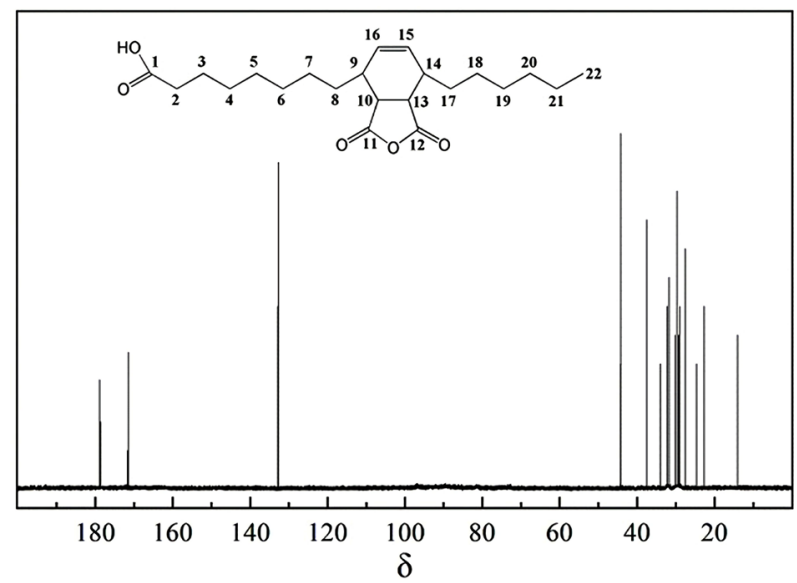

Figure $5 .{ }^{13} \mathrm{C}$ NMR spectrum of MAC.

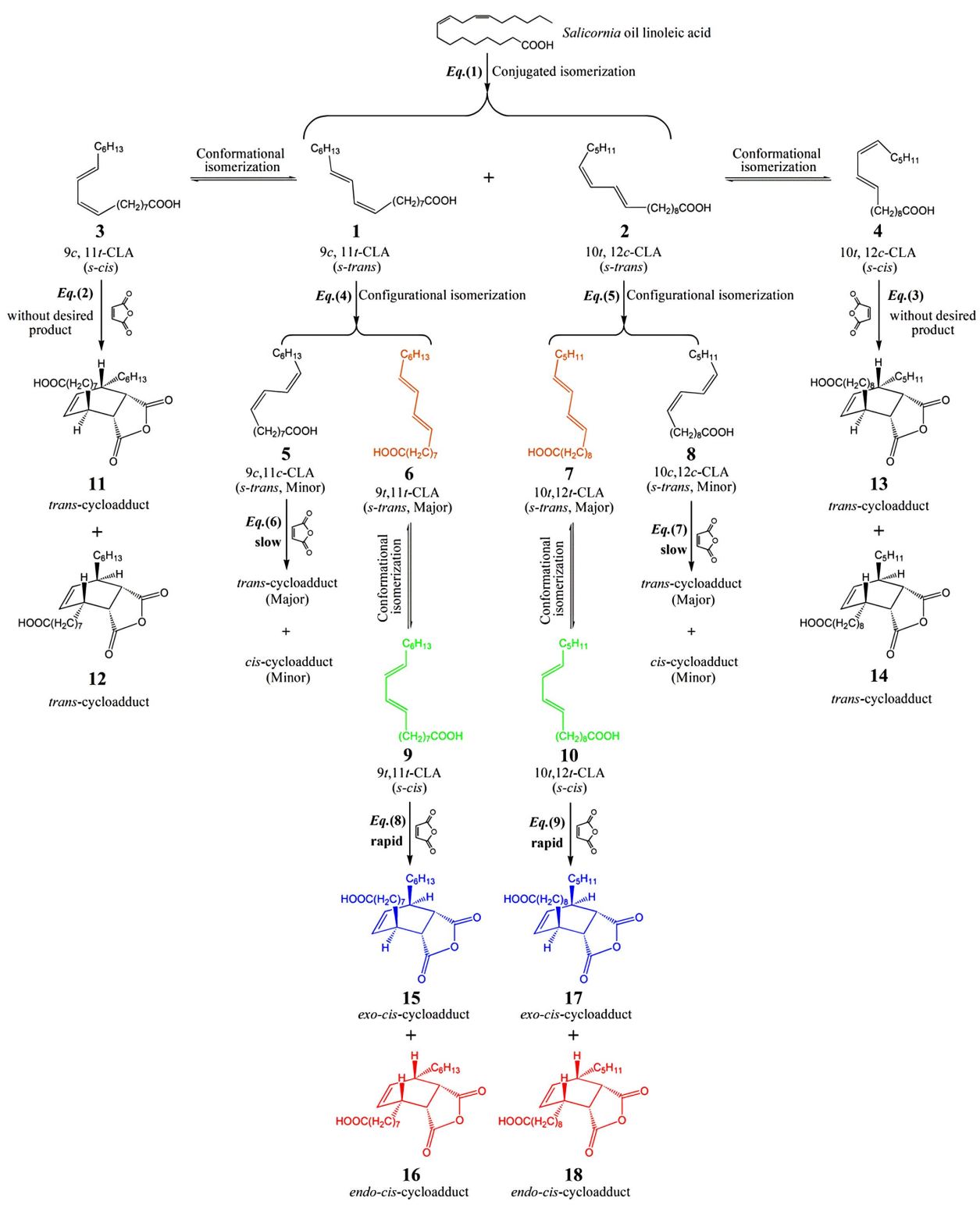

Scheme 1. Proposed stereochemical mechanism for the improved preparation of MAC. 
Researches on the Diels-Alder reactions of simple conjugated dienes with maleic anhydride have been intensively concerned, allowing us to predict the reactions involving more complex conjugated dienes, such as CLA. Conjugated dienes can exist in two different planar conformations: an $s$-trans conformation and an $s$-cis conformation. $S$-trans means that the conjugated double bonds are trans about the single bond ( $s=$ single); while $s$-cis means that the conjugated double bonds are cis about the single bond. As far as we know, even in the same configuration, CLA can exist in many different conformations due to the free rotation of the single C-C bond. Hence, it is very critical for CLA conformer to display a more favorable geometric structure to react with maleic anhydride more appropriately. To further confirm this hypothesis, the influence of structural parameters of CLA conformers on the stereochemical reactions was firstly investigated by Mopac- $\mathrm{AM}_{1}$ algorithm in Chem3D Ultra 10.0.

\subsubsection{Structural Parameters of CLA and MAC Conformers}

The Mopac- $\mathrm{AM}_{1}$ algorithm in Chem3D Ultra 10.0 was employed to optimize the geometric structures of CLA and MAC conformers. This method was operated as follows: 1) draw 3D molecular structural models of different conformers by Chem3D Ultra 10.0; 2) start two "Minimize Energy" procedures successively in "MM2" and "Mopac interface" module on "Calculations" menu to alternatively optimize the geometric structure of these conformers till the standard formation enthalpy $\left(\Delta_{f} H_{m}^{\theta}\right.$ ) remained almost unchanged (the gap between two $\Delta_{f} H_{m}^{\theta} \mathrm{s}$ from adjacent optimizations was less than $\left.0.1 \mathrm{Kcal} \cdot \mathrm{mol}^{-1}\right)$; and 3) calculate quantum-chemical parameters of the optimized conformers by Mopac- $\mathrm{AM}_{1}$ algorithm. The optimized geometric structures of CLA and MAC conformers derived from conformer 1 (i.e., $9 c, 11 t$-CLA) could be obtained (Figure 6). And the theoretical structural parameters of the conjugated dienes of CLA before and after cycloaddition reaction are also shown (Table 1 ).

It is obvious that no significant difference in both bond angles and bond lengths could be observed for CLA conformers or MAC conformers (Table 1). However,

Table 1. A comparison of theoretical structural parameters of the conjugated dienes before (in CLA) and after (in MAC) cycloaddition reaction.

\begin{tabular}{|c|c|c|c|c|c|c|c|c|c|}
\hline \multirow{2}{*}{\multicolumn{3}{|c|}{ Conformer }} & \multicolumn{2}{|c|}{ Bond angle $/^{\circ}$} & \multicolumn{3}{|c|}{ Bond length/A } & \multirow{2}{*}{$\begin{array}{c}\text { Dihedral angle } /^{\circ} \\
\mathrm{C}_{9}-\mathrm{C}_{10}-\mathrm{C}_{11}-\mathrm{C}_{12}\end{array}$} & \multirow{2}{*}{$\begin{array}{c}\mathrm{C}_{9} \leftrightarrow \mathrm{C}_{12} \\
\text { distance } / \AA\end{array}$} \\
\hline & & & $\mathrm{C}_{9}-\mathrm{C}_{10}-\mathrm{C}_{11}$ & $\mathrm{C}_{10}-\mathrm{C}_{11}-\mathrm{C}_{12}$ & $\mathrm{C}_{9}-\mathrm{C}_{10}$ & $\mathrm{C}_{10}-\mathrm{C}_{11}$ & $\mathrm{C}_{11}-\mathrm{C}_{12}$ & & \\
\hline \multirow{5}{*}{ 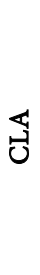 } & 1 & $s$-trans & 124.8 & 123.0 & 1.341 & 1.448 & 1.341 & -179.7 & 3.689 \\
\hline & 3 & $s$-cis & 124.7 & 122.8 & 1.341 & 1.448 & 1.340 & 179.7 & 3.687 \\
\hline & 5 & $s$-trans & 124.8 & 124.7 & 1.341 & 1.447 & 1.341 & -179.0 & 3.703 \\
\hline & 6 & $s$-trans & 123.1 & 123.0 & 1.340 & 1.448 & 1.341 & 179.9 & 3.677 \\
\hline & 9 & $s-c i s$ & 125.1 & 125.4 & 1.340 & 1.447 & 1.341 & -15.0 & 3.010 \\
\hline \multirow{2}{*}{ 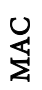 } & 15 & exo-cis & 120.7 & 121.7 & 1.490 & 1.336 & 1.491 & 1.5 & 2.880 \\
\hline & 16 & endo-cis & 119.6 & 119.5 & 1.491 & 1.338 & 1.491 & 0.0 & 2.809 \\
\hline
\end{tabular}




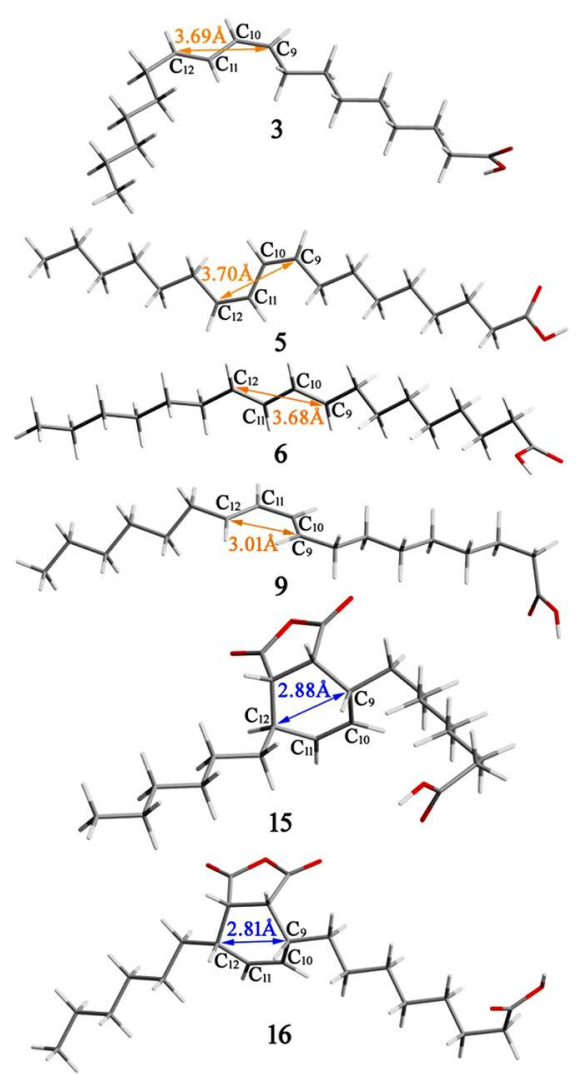

Figure 6. Optimized geometric structures of CLA and corresponding MAC conformers.

bond angles of CLA conformers were larger than those of MAC conformers. In addition, $\mathrm{C}_{9}-\mathrm{C}_{10}$ and $\mathrm{C}_{11}-\mathrm{C}_{12}$ bond lengths of CLA conformers were shorter than those of MAC conformers, whereas $\mathrm{C}_{10}-\mathrm{C}_{11}$ bond of CLA conformers were relatively longer. We can speculate that the cyclohexenyl ring formation between maleic anhydride and conjugated dienes of CLA was responsible for the decrease in bond angles of MAC conformers. The stronger electron-withdrawing effect of dienophile (i.e., maleic anhydride) would resulted in the increase in $\mathrm{C}_{9}-\mathrm{C}_{10}$ and $\mathrm{C}_{11}-\mathrm{C}_{12}$ bond lengths of MAC conformers, and the transformation of $\mathrm{C}_{10}-\mathrm{C}_{11}$ bond geometry from $\mathrm{sp}^{3}-\mathrm{sp}^{3}$ hybridization (in CLA) into $\mathrm{sp}^{2}-\mathrm{sp}^{2}$ hybridization (in MAC) could lead to the decrease in $\mathrm{C}_{10}-\mathrm{C}_{11}$ bond lengths of MAC conformers. Normally, short bond will be formed if the $s$-character of a hybridized orbital increases. Obviously, greater proportion of $s$-character could be observed in the $\mathrm{sp}^{2}$ hybridization than in the $\mathrm{sp}^{3}$ hybridization.

Noticing that $\mathrm{C}_{9}-\mathrm{C}_{10}-\mathrm{C}_{11}-\mathrm{C}_{12}$ dihedral angle and $\mathrm{C}_{9} \leftrightarrow \mathrm{C}_{12}$ distance of conformer 9 were distinctly different from those of other four CLA conformers (i.e., conformer 1, 3, 5 and 6), while the values of conformer 9 were close to those of MAC conformers (i.e., conformer 15 and 16). Although conformer 3 had the same $s$-cis conformation as conformer 9 , the values of $\mathrm{C}_{9}-\mathrm{C}_{10}-\mathrm{C}_{11}-\mathrm{C}_{12}$ dihedral angle and $\mathrm{C}_{9} \leftrightarrow \mathrm{C}_{12}$ distance of the latter were much closer to those of MAC conformers than the former. Therefore, among all the CLA conformers derived from conformer 1 (i.e., $9 c, 11$-CLA), only conformer 9 displayed the most favorable 
structural parameters for ring formation.

Similarly, through the comparison of structural parameters of other five CLA conformers (i.e., conformer 2, 4, 7, 8 and 10) with those of corresponding MAC conformers (data not shown), we could find that among all the CLA conformers derived from conformer 2 (i.e., $10 t, 12 c$-CLA), conformer 10 exhibited more favorable structural parameters for ring formation, which was similar to conformer 9.

\subsubsection{Establishment of Conformers-Ratio Calculation Method}

To deeply study the stereochemical mechanism for the preparation of MAC, we also developed a new method for calculating conformers-ratio (i.e., $N_{i}: N_{j}$ ) of CLA or MAC. The calculation method can be defined as Expression (1).

$$
\frac{N_{i}}{N_{j}}=\exp \left[\left(\Delta_{f} H_{m, j}^{\theta}-\Delta_{f} H_{m, i}^{\theta}\right) / R T\right]
$$

where $N_{i}$ and $N_{j}$ are the numbers of conformer $i$ and $j$, respectively; $\Delta_{f} H_{m, j}^{\theta}$ and $\Delta_{f} H_{m, i}^{\theta}\left(\mathrm{J} \cdot \mathrm{mol}^{-1}\right)$ are the standard formation enthalpies of conformer $i$ and $j$, respectively; $T(\mathrm{~K})$ is the equilibrium temperature of reaction system (i.e., 333.15 $\mathrm{K}$, see 3.1$)$; and $R\left(\mathrm{~J} \cdot \mathrm{mol}^{-1} \cdot \mathrm{K}^{-1}\right)$ is the Boltzmann constant.

A derivation of Expression (1) could be obtained. According to Maxwell-Boltzmann statistics, the conformers-ratio $\left(N_{i}: N_{j}\right)$ under thermodynamic equilibrium can be derived as Expression (2).

$$
\frac{N_{i}}{N_{j}}=\frac{\exp \left(-E_{i} / R T\right)}{\exp \left(-E_{j} / R T\right)}=\exp \left[\left(E_{j}-E_{i}\right) / R T\right]
$$

where $E_{i}$ and $E_{j}\left(\mathrm{~J} \cdot \mathrm{mol}^{-1}\right)$ are the energies of conformer $i$ and $j$, respectively; $T(\mathrm{~K})$ is the equilibrium temperature of the system; and $R\left(\mathrm{~J} \cdot \mathrm{mol}^{-1} \cdot \mathrm{K}^{-1}\right)$ is the Boltzmann constant. We also tried to find the relations between $E_{i}$ and $\Delta_{f} H_{m, i}^{\theta}$, as well as the relations between $E_{j}$ and $\Delta_{f} H_{m, j}^{\theta}$. According to the thermodynamic definition of standard formation enthalpy, $E_{i}$ and $E_{j}$ in Expression (2) can be calculated by Expression (3) and (4), respectively.

$$
\begin{gathered}
\Delta_{f} H_{m, i}^{\theta}=E_{i}-\left(E_{\mathrm{C}}+E_{\mathrm{O}_{2}}+E_{\mathrm{H}_{2}}\right) \\
\Delta_{f} H_{m, j}^{\theta}=E_{j}-\left(E_{\mathrm{C}}+E_{\mathrm{O}_{2}}+E_{\mathrm{H}_{2}}\right)
\end{gathered}
$$

where $E_{\mathrm{C}}, E_{\mathrm{O}_{2}}$ and $E_{\mathrm{H}_{2}}\left(\mathrm{~J} \cdot \mathrm{mol}^{-1}\right)$ are the energies of carbon, oxygen gas and hydrogen gas, respectively. When both $i$ and $j$ are CLA or MAC isomers, $i$ has the same value of $\left(E_{\mathrm{C}}+E_{\mathrm{O}_{2}}+E_{\mathrm{H}_{2}}\right)$ as $j$. Thereby, we can derive Expression (5) from Expression (3) and (4). Finally, Expression (2) can be rewritten into Expression (1) in terms of Expression (5).

$$
E_{j}-E_{i}=\Delta_{f} H_{m, j}^{\theta}-\Delta_{f} H_{m, i}^{\theta}
$$

\subsubsection{Conformers-Ratios, FMO Energy Levels and $\Delta_{f} H_{m}^{\theta} s$ of CLA and MAC}

Based on the determined geometric structures of CLA and MAC conformers 
(see 3.6.2), we could investigate the influences of electron-donor abilities and stabilities of CLA conformers on the stereochemical reactions of Scheme 1. FMO theory assumes that chemical reactivity correlates with $E_{\mathrm{HOMO}}$ (energy of the highest occupied molecule orbital) and $E_{\mathrm{LUMO}}$ (energy of the lowest unoccupied molecule orbital). The conformer with higher $E_{\text {Номо }}$ should is a better electrondonor, while the conformer with lower $E_{\mathrm{LUMO}}$ should be a better electron-acceptor; and a higher electron-donor ability of CLA conformer will greatly facilitate the cycloaddition reaction of maleic anhydride. The energy gap $\Delta E$ (i.e., $\left.E_{\mathrm{LUMO}}-E_{\mathrm{HOMO}}\right)$ reflects the chemical stability of a molecule; and the lower the $\Delta E$ value is, the more easily the electron transition occurs, and the higher the chemical reactivity is. In addition, as a measure for the stability of molecular structure, the more negative the $\Delta_{f} H_{m}^{\theta}$ value is, the more stable the conformer would be, and consequently the more favorable the formation reaction is.

In order to investigate the influences of electron-donor abilities and stabilities of CLA conformers on the stereochemical reactions, three parameters (i.e., $E_{\mathrm{HOMO}}$, $E_{\mathrm{LUMO}}$ and $\Delta_{f} H_{m}^{\theta}$ ) of all CLA conformers and MAC conformers were exported from Chem3D Ultra 10.0 (Table 2). Based on the data of $\Delta_{f} H_{m}^{\theta} \mathrm{s}$, the conformers-ratios of CLA and MAC were calculated by Expression (1). The calculated results, together with their $\Delta E$ s, are also shown in Table 2.

Table 2. Conformers-ratios, FMO energy levels and $\Delta_{f} H_{m}^{\theta} \mathrm{s}$ of CLA and MAC conformers.

\begin{tabular}{|c|c|c|c|c|c|c|c|c|}
\hline & \multicolumn{2}{|c|}{ Conformer } & \multirow{3}{*}{$\begin{array}{c}E_{\mathrm{HOMO}} / \mathrm{eV} \\
-8.848 \\
-8.821\end{array}$} & \multirow{3}{*}{$\begin{array}{c}E_{\mathrm{LUMO}} / \mathrm{eV} \\
0.361 \\
0.390\end{array}$} & \multirow{3}{*}{$\begin{array}{c}\Delta E / \mathrm{eV} \\
9.209 \\
9.211\end{array}$} & \multirow{3}{*}{$\begin{array}{c}\Delta_{f} H_{m}^{\theta} / \mathbf{k J} \cdot \mathrm{mol}^{-1} \\
-652.06 \\
-649.59\end{array}$} & \multirow{3}{*}{$\begin{array}{c}\text { Conformers-ratio } \\
N_{2}: N_{1}=0.52: 1 \\
\left(N_{1} \%=65.79 w \%\right)\end{array}$} & \multirow{3}{*}{$\begin{array}{c}\text { Reaction equation } \\
\text { Equation (1) }\end{array}$} \\
\hline \multirow{11}{*}{ 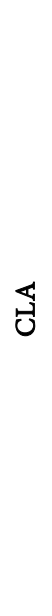 } & ฐิี & 1 & & & & & & \\
\hline & $\begin{array}{l}\vec{z} \\
\dot{b}\end{array}$ & 2 & & & & & & \\
\hline & 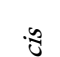 & 3 & -8.886 & 0.315 & 9.201 & -625.42 & $N_{3}: N_{1}=6.7 \times 10^{-5}: 1$ & Conformational \\
\hline & ¿ & 4 & -8.797 & 0.403 & 9.200 & -646.37 & $N_{4}: N_{2}=0.31: 1$ & isomerization \\
\hline & \multirow{4}{*}{$\begin{array}{l}\text { ב } \\
\text { İ } \\
\dot{b}\end{array}$} & 5 & -8.848 & 0.352 & 9.200 & -648.57 & \multirow{2}{*}{$N_{6}: N_{5}=8.06: 1$} & \multirow{2}{*}{ Equation (4) } \\
\hline & & 6 & -8.854 & 0.362 & 9.216 & -654.35 & & \\
\hline & & 7 & -8.844 & 0.372 & 9.216 & -654.37 & \multirow[b]{2}{*}{$N_{7}: N_{8}=19.45: 1$} & \multirow{2}{*}{ Equation (5) } \\
\hline & & 8 & -8.819 & 0.379 & 9.198 & -646.15 & & \\
\hline & \multirow{3}{*}{$\begin{array}{l}3 \\
\dot{b}\end{array}$} & 9 & -8852 & 0.454 & 9306 & -64639 & $N_{9}: N_{3}=1941: 1$ & Conformational \\
\hline & & 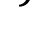 & -0.052 & 0.404 & 5.000 & & $N_{9}: N_{5}=0.46: 1$ & isomerization \\
\hline & & 10 & -8.883 & 0.435 & 9.318 & -647.80 & $N_{10}: N_{4}=1.68: 1 \quad N_{10}: N_{8}=1.81: 1$ & (predominant reactants) \\
\hline \multirow{8}{*}{ 岁 } & \multirow{4}{*}{ 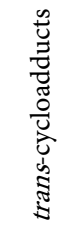 } & 11 & -10.303 & 0.272 & 10.575 & -1163.94 & \multirow{2}{*}{ without desired product } & \multirow{2}{*}{ Equation (2) } \\
\hline & & 12 & -10.282 & 0.275 & 10.557 & -1164.48 & & \\
\hline & & 13 & -10.289 & 0.278 & 10.567 & -1163.76 & \multirow{2}{*}{ without desired product } & \multirow[t]{2}{*}{ Equation (3) } \\
\hline & & 14 & -10.296 & 0.264 & 10.560 & -1166.49 & & \\
\hline & \multirow{4}{*}{ 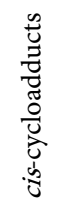 } & 15 & -10.228 & 0.294 & 10.522 & -1162.10 & \multirow{2}{*}{$N_{16}: N_{15}=2.14: 1$} & \multirow{2}{*}{$\begin{array}{c}\text { Equation (8) } \\
\text { (main reaction) }\end{array}$} \\
\hline & & 16 & -10.303 & 0.271 & 10.574 & -1164.21 & & \\
\hline & & 17 & -10.270 & 0.269 & 10.539 & -1164.30 & \multirow{2}{*}{$N_{18}: N_{17}=1.99: 1$} & \multirow{2}{*}{$\begin{array}{c}\text { Equation (9) } \\
\text { (main reaction) }\end{array}$} \\
\hline & & 18 & -10.316 & 0.261 & 10.577 & -1166.20 & & \\
\hline
\end{tabular}


It can be seen from Table 2 that by Expression (1), the calculated value of the weight percentage of conformer 1 in as-prepared CLA (i.e., $65.79 w t \%$ ) was excellently consistent with the experimental value in this work (i.e., $63.82 w t \%$, as shown in 2.2), which was also in accordance with the results previously reported [15], indicating that Expression (1) possessed acceptability and accuracy for estimating conformers-ratio under thermodynamic equilibrium.

It was almost impossible for Diels-Alder reaction Equation (2) to be carried out because the percentage of conformer 3 was really low (trace level, $N_{3}: N_{1}=6.7 \times 10^{-5}: 1$ ); and the very low percentage of conformer 4 originating from the less $N_{2}: N_{1}$, and especially $\mathrm{N}_{4}: \mathrm{N}_{2}$, was very unfavorable for Diels-Alder reaction Equation (3). In comparison with conformer 9 and 10, conformer 3 and 4 had less negative $\Delta_{f} H_{m}^{\theta} \mathrm{s}$, indicating that the former two were more stable than the latter two $\left(N_{9}: N_{3}=1941: 1 ; N_{10}: N_{4}=1.68: 1\right)$. Obviously, it would become much more difficult for Equation (2) and Equation (3) to be carried out, particularly in the presence of iodine catalyst which was more preferential for the formation of conformer $\mathbf{9}$ and $\mathbf{1 0}$ rather than conformer $\mathbf{3}$ and $\mathbf{4}$. More importantly, conformer 3 and 4 displayed more unfavorable geometric structures for ring formation in comparison with conformer 9 and 10 (see Table 1). Therefore, Diels-Alder reaction Equation (2) and Equation (3) could hardly occur; and the results obtained from the calculated conformers-ratios and the $\Delta_{f} H_{m}^{\theta} \mathrm{s}$, were in excellent agreement with those from the experiments in this work, e.g., no desired product could be obtained in the absence of iodine catalyst (see 3.2).

It is remarkable that $\Delta_{f} H_{m}^{\theta}$ s of conformer 6 and 7 were the most negative among all the studied CLA conformers, indicating that these two conformers were the most stable structures. That is to say, Equation (4) and Equation (5) could be successfully carried out in the presence of the catalyst, iodine. Therefore, conformer 6 and 7 were determined as the predominant products of Equation (4) and Equation (5), respectively. According to the corresponding conformers-ratios, it is obvious that conformer $\mathbf{5}$ and $\mathbf{8}$ were the by-products of Equation (4) and Equation (5), respectively (Table 2).

No remarkable differences among six $E_{\mathrm{HOMO}} \mathrm{s}$ of conformers 5 to 10 could be observed. In comparison with that of conformer $6, E_{\mathrm{HOMO}}$ of conformer 9 was a little less negative, indicating that the latter possessed slightly higher electrondonor ability than that of the former. Simultaneously, conformer 9 had higher $E_{\mathrm{LUMO}}$ in comparison with conformer 6 , which indicated that the electron-accepting ability of the former was inferior to that of the latter. In terms of Lewis acid-base theory, this also means that conformer 9 might possess superior electron-donor ability than conformer 6 , which could facilitate the cycloaddition reaction between conformer 9 and maleic anhydride.

Although the ratio of conformer 9 to conformer 5 was a little low ( $N_{9}: N_{5}=0.46: 1$ ), the former did act as a predominant reactant for Equation (8), which could be ascribed to the following factors. Firstly, conformer 6, instead of conformer 5, was determined as the predominant product of Equation (4) ( $\left.N_{6}: N_{5}=8.06: 1\right)$. Secondly, the conformational transformation of confor- 
mer 6 into conformer 9 could be easily carried out because Equation (8) was a synergistic and rapid reaction that could occurred in one-step without appearance of any active intermediate. More importantly, among all studied CLA conformers derived from conformer 1, conformer 9 rather than conformer 6 had the most favorable structural parameters for ring formation. Therefore, conformer 9 was the predominant reactant for Diels-Alder reaction Equation (8), which was the main reaction in the preparation of MAC; while Equation (6) was a side reaction since conformer 5 with low percentage $\left(N_{5}: N_{6}=1: 8.06\right)$ acted as the reactant for Equation (6), and in comparison with conformer 9, conformer $\mathbf{5}$ was also significantly inferior for ring formation due to its geometric structure.

Similarly, we could deduce that conformer 10 was the predominant reactant for Diels-Alder reaction Equation (9) that was another main reaction in the preparation of MAC. Due to the existence of two different isomers (i.e., $9 c, 11 t$ and $10 t, 12 c)$ in as-prepared CLA, the two main reactions were therefore involved in the preparation of MAC. In comparison with conformer $\mathbf{9}$, conformer 10 showed another advantage for acting as the predominant reactant, such as the value of $N_{10}: N_{8}(=1.81)$ was much greater than that of $N_{9}: N_{5}(=0.46)$. In addition, it is obvious that Equation (7) was another side reaction in the preparation of MAC, which was similar to Equation (6).

According to FMO theory, the cycloaddition reaction of Equation (8) occurred between HOMO of conformer 9 and LUMO of maleic anhydride, where the bonding electrons excited from the HOMO level into the LUMO level. The products of Equation (8) (i.e., conformer 15 and 16) were determined to be cis-cycloadducts that displayed exo and endo conformations, respectively, which might be due to the fact that conformer 9 was in a relatively stable $s$-cis conformation, and Equation (8) was a stereospecific cis-addition reaction. Similarly, other two cis-cycloadduct products with respectively exo and endo conformations (i.e., conformer 17 and 18) could be obtained from Equation (9). As shown in Table 2, cis-cycloadducts were determined as main products in the preparation of MAC; and the endo/exo ratios of the cis-cycloadducts derived from $9 c$, $11 t$ - and $10 t, 12 c$-CLA, were calculated to be $2.14: 1$ and $1.99: 1$, respectively, by Expression (1). Obviously, higher percentage of endo conformation rather than exo conformation could be formed, even though the former was more sterically congested and less thermodynamically stable. This could be attributed to the fact that the endo conformation was stabilized by $\pi$ orbital overlap of the $\mathrm{C}=\mathrm{O}$ groups in maleic anhydride with the HOMO of conformer 9 or conformer 10 , i.e., so-called "secondary orbital overlap" [12].

Although $\Delta_{f} H_{m}^{\theta}$ s of trans-cycloadducts (e.g., conformer 11, 12 and 14) were a little more negative than those of cis-cycloadducts (e.g., conformer 15, 16 and 18), no desired products would be obtained via Equation (2) and Equation (3) as explained previously. Even if trans-cycloadduct was probably formed from Equation (6) or Equation (7), its percentage was much lower than that of cis-cycloadduct since Equation (6) and Equation (7) were merely side reactions. Therefore, 
Table 3. Conformational nomenclatures for the prepared MAC.

\begin{tabular}{cc}
\hline MAC & Nomenclature \\
\hline Conformer 15 & Exo-cis-8-[(3aS,4S,7R,7aR)-7-hexyl-1,3-dioxo-3a,4,7,7a-tetrahydro-2-benzofuran-4-yl]-octanoic acid \\
Conformer 16 & Endo-cis-8-[(3aS,4S,7R,7aR)-7-hexyl-1,3-dioxo-3a,4,7,7a-tetrahydro-2-benzofuran-4-yl]-octanoic acid \\
Conformer 17 & Exo-cis-9-[(3aS,4S,7R,7aR)-7-pentyl-1,3-dioxo-3a,4,7,7a-tetrahydro-2-benzofuran-4-yl]-pelargonic acid \\
Conformer 18 & Endo-cis-9-[(3aS,4S,7R,7aR)-7-pentyl-1,3-dioxo-3a,4,7,7a-tetrahydro-2-benzofuran-4-yl]-pelargonic acid \\
\hline
\end{tabular}

trans-cycloadduct was obtained only as by-product in the preparation of MAC.

Based on the description and discussion of the above, the stereochemical mechanism for the improved preparation of MAC could be proposed as Scheme 1.

\subsubsection{Conformational Nomenclatures of MAC}

According to the systematic nomenclature, the four conformers of prepared MAC were named (Table 3).

\section{Conclusions}

By the improved process described in this article, MAC was prepared in a high yield of $96.7 \%$, which can provide important references for industrial production of MAC. The new calculation method that possessed acceptability and accuracy for estimating conformers-ratio of CLA or MAC was established, and mainly by this calculation method, the stereochemical mechanism for the improved preparation of MAC was proposed, which can function as a theoretical basis for the determination and separation of related conformers. And the following conclusions were also obtained:

1) The catalytic configurational isomerization of CLA, as well as Diels-Alder $[4+2]$ cycloaddition of the isomerized CLA with maleic anhydride, were mainly involved and occurred simultaneously in one step for the preparation of MAC.

2) It was confirmed that the cycloaddition occurred mainly between maleic anhydride and trans-trans CLA, rather than between maleic anhydride and cis-trans or trans-cis CLA. From all studied CLA conformers, the most stable one was the $s$-trans conformation of trans-trans CLA, while its $s$-cis conformation had the most favorable structural parameters for cyclohexenyl ring formation.

3) The trans-trans CLA in the $s$-cis conformation acted as a predominant reactant for the cycloaddition of maleic anhydride, which was confirmed as the main reaction in the improved process.

4) Four MAC conformers derived from $9 c, 11 t$ - and $10 t, 12 c$-CLA, were obtained as final main products and determined to be cis-cycloadducts, which were also named according to the systematic nomenclature. And the endo/ exo ratios of the cis-cycloadducts derived from $9 c, 11 t$ - and $10 t, 12 c$-CLA, were calculated to be $2.14: 1$ and 1.99:1, respectively.

5) The results obtained by the calculation method were in good consistent with those of our experiments. 


\section{Acknowledgements}

The authors are grateful for financial supports from the National Natural Science Foundation of China (No. 21762016) and the Innovation \& Entrepreneurship Training Project for College Students in Hainan Province (No. S201911100030).

\section{Conflicts of Interest}

The authors declare no conflicts of interest regarding the publication of this paper.

\section{References}

[1] Sullivan, M.J., Binette, M.L. and Aoyama, S. (2019) Two-Piece Golf Ball Comprising Highly Neutralized Polymer Layer. Acushnet Company, New Bedford.

[2] Huang, K., Zhang, P., Zhang, J.W., Li, S.H., Li, M., Xia, J.L. and Zhou, Y.H. (2013) Preparation of Biobased Epoxies Using Tung Oil Fatty Acid-Derived $\mathrm{C}_{21}$ Diacid and $\mathrm{C}_{22}$ Triacid and Study of Epoxy Properties. Green Chemistry, 15, 2466-2475. https://doi.org/10.1039/c3gc40622a

[3] Ursula, B., Anton, J. and Juergen, O.M. (2012) Esters of Maleinized Fatty Compounds as Plasticizers. European Journal of Lipid Science and Technology, 114, 49-54. https://doi.org/10.1002/ejlt.201100136

[4] Watanabe, S., Fujita, T., Fukuda, S., Hirano, K. and Sakamoto, M. (1986) Characteristic Properties as Cutting Fluid Additives of the Products from the Reaction of Unsaturated Fatty Acids with Maleic Anhydride. Materials Chemistry and Physics, 15, 89-96. https://doi.org/10.1016/0254-0584(86)90091-X

[5] Moreno, M., Victoria Gomez, M., Cebrian, C., Prieto, P., Hoza, A. and Moreno, A. (2012) Sustainable and Efficient Methodology for CLA Synthesis and Identification. Green Chemistry, 14, 2584-2594. https://doi.org/10.1039/c2gc35792e

[6] Ojha, D.P. (2005) Ordering of a Thermotropic Mesogen at Phase Transition Temperature-A Statistical Approach Based on Quantum Mechanics. Journal of Theoretical \& Computational Chemistry, 4, 803-810. https://doi.org/10.1142/S0219633605001805

[7] Hartung, J., Daniel, K., Rummey, C. and Bringmann, G. (2006) On the Stereoselectivity of 4-penten-1-oxyl Radical 5-exo-trig Cyclizations. Organic \& Biomolecular Chemistry, 4, 4089-4100. https://doi.org/10.1039/b611473c

[8] Jin, P., Yang, L., Liu, C., Chen, M. Q., Hou, Q. H., Li, L. L. and Zhao, Y. J. (2017) A Comparative Study on the N-Heterocyclic Carbene Adducts of $I_{\mathrm{h}}-\mathrm{C}_{60}, \mathrm{D}_{5 \mathrm{~h}}-\mathrm{C}_{70}$ and Sc3N@I $\mathrm{I}_{\mathrm{h}}-\mathrm{C}_{80}$. Physical Chemistry Chemical Physics, 19, 17598-17606. https://doi.org/10.1039/C7CP02696)

[9] Liu, Q., Chen, C.J., Zhang, L. and Gao, K. (2016) Two New Indole Alkaloids from Hunteria Zeylanica. Journal of Asian Natural Products Research, 18, 349-353. https://doi.org/10.1080/10286020.2015.1092961

[10] El Merbouh, B., Bourjila, M., Tijar, R., El Bouzaidi, R.D., El Gridani, A. and El Mouhtadi, M. (2014) Conformational Space Analysis of Neutral and Protonated Glycine Using a Genetic Algorithm for Multi-Modal Search. Journal of Theoretical \& Computational chemistry, 13, Article No. 1450067. https://doi.org/10.1142/S0219633614500679

[11] Chin, S.F., Liu, W., Storkson, J.M., Ha, Y.L. and Pariza, M.W. (1992) Dietary Sources of Conjugated Dienoic Isomers of Linoleic Acid, a Newly Recognized Class 
of Anticarcinogens. Journal of Food Composition and Analysis, 5, 185-197. https://doi.org/10.1016/0889-1575(92)90037-K

[12] Arrieta, A., Cossio, F.P. and Lecea, B. (2001) Direct Evaluation of Secondary Orbital Interactions in the Diels-Alder Reaction between Cyclopentadiene and Maleic Anhydride. Journal of Organic Chemistry, 66, 6178-6180.

https://doi.org/10.1021/jo0158478

[13] Eulitz,K., Yurawecz, M.P., Sehat, N., Fritsche, J., Roach, J.A.G., Mossoba, M.M., Kramer, J.K.G., Adlof, R.O. and Ku, Y. (1999) Preparation, Separation and Confirmation of the Eight Geometrical cis/trans Conjugated Linoleic Acid Isomers 8,10through 11,13-18:2. Lipids, 34, 873-877.

https://doi.org/10.1007/s11745-999-0435-Z

[14] Arca, M., Sharma, B.K., Price, N.P.J., Perez, J.M. and Doll, K.M. (2012) Evidence Contrary to the Accepted Diels-Alder Mechanism in the Thermal Modification of Vegetable Oil. Journal of the American Oil Chemists Society, 89, 987-994. https://doi.org/10.1007/s11746-011-2002-x

[15] Wu, Y., Yang, R.Q., Chen, M.Z. and Liao, X.B. (2012) Antitumor Activity of Conjugated Linoleic Acid Converted from Linoleic Acid in Salicornia herbacea Seed Oil. Food Science, 33, 318-322. (In Chinese) 